\title{
Structure and expression of a Drosophila male accessory gland gene whose product resembles a peptide pheromone precursor
}

\author{
Scott A. Monsma ${ }^{1}$ and Mariana F. Wolfner ${ }^{2,3}$ \\ ${ }^{1}$ Section of Biochemistry, Molecular, and Cell Biology and ${ }^{2}$ Section of Genetics and Development, Cornell University, Ithaca, \\ New York 14853 USA
}

\begin{abstract}
The accessory gland of male insects is a genital tissue that secretes many components of the ejaculatory fluid, some of which affect the female's receptivity to courtship and her rate of oviposition. We have examined the structure and expression of two tightly linked genes that are expressed exclusively in the male accessory glands of adult Drosophila melanogaster. The two genes are transcribed from the same strand of DNA, and are separated by 20 bases. Both genes are regulated by the sex determination hierarchy and are expressed in the absence of germ cells. Immunological analysis reveals the protein products of at least one of these genes in the secretion of the accessory gland. The proteins are transferred to the female fly during copulation and are rapidly altered in the female genital tract. The predicted sequence of one protein has features of a peptide hormone precursor, and a region in which 11 of 17 amino acids are identical to egg-laying hormone (ELH) of the California sea hare, Aplysia californica.
\end{abstract}

[Key Words: Drosophila; accessory gland; Aplysia ELH; peptide pheromone; sex determination]

Received May 31, 1988; revised version accepted July 18, 1988.

In this study we describe genes that are expressed in the accessory gland, a male tissue whose secreted products mediate stereotyped behavioral and physiological changes in female Drosophila melanogaster after mating. Such behavioral changes include a decrease in female receptivity to courtship by males (evidenced by female avoidance of courting males, Manning 1962), and increased egg-laying behavior (oviposition) (Leopold 1976; Chen 1984). Physiological changes in the ovary are correlated with the increase in oviposition, such that the rate of egg production is doubled relative to unmated females (Fowler 1973; Chen 1984). Eggs laid by mated females are also larger than the sterile eggs laid by unmated females (Fowler 1973). These behavioral and physiological effects persist for long periods after mating: the decrease in receptivity lasts as long as 7-9 days (Manning 1962), while the enhancement of oviposition lasts about 14 days (Chen and Bühler 1970).

Some of these post-copulatory effects are induced by the secretions of the male accessory glands (paragonia), which are introduced into the female during mating (Chen 1984). Implantation of the male accessory gland (Garcia-Bellido 1964) or injection of its secretion into virgin females (Chen and Bühler 1970; Baumann 1974b) causes decreased receptivity to courtship and stimulation of egg production by the ovary. Accessory gland secretions may also be involved in sperm storage and utili-

${ }^{3}$ Corresponding author. zation (Fowler 1973). The accessory gland secretion is a complex mixture of free amino acids and amino acid derivatives, proteins, lipids, carbohydrates, and large (visible) aggregates and vesicles (Federer and Chen 1982). The complexity of the secretion and the difficulty of collecting sufficient material has hindered the purification and analysis of specific components that might be involved in eliciting these behavioral and physiological effects.

Several studies have examined the protein components of the accessory gland secretion using one- or twodimensional gel electrophoresis (Ingman-Baker and Candido 1980; Stumm-Zollinger and Chen 1985; Whalen and Wilson 1986); considerable protein heterogeneity is seen between species (Chen et al. 1985). In D. melanogaster, certain polymorphic protein bands have been correlated with specific chromosomal regions (Whalen and Wilson 1986); however, the functions of these proteins are unknown.

Two components have been purified from the male accessory gland secretion of Drosophila funebris (Baumann 1974a). PS-1, a peptide of 27 amino acids, causes a decrease in receptivity of unmated females to male courtship. PS-2, a glycine-carbohydrate derivative, causes an increase in oviposition when injected into unmated females (Baumann 1974b). Neither isolated component elicits the effect as strongly as unfractionated secretion, nor does either component elicit a detectable effect when injected into female $D$. melanogaster 
(Baumann 1974b). The genes that are involved in the production of these two components are unknown, although the amino acid sequence of the peptide has been determined (Baumann et al. 1975).

We have taken a molecular genetic approach to accessory gland function: cloning genes that are expressed specifically in the accessory gland, to examine their structure and expression with the goal of defining the function of their products. Here, we report the structure and expression of two genes that encode secretory proteins of the male accessory gland of $D$. melanogaster.

\section{Results}

mst 355a and mst 355b: male-specific transcripts

We initially identified a restriction fragment encoding male-specific transcripts in screens of a genomic walk (MacIntyre and Davis 1987) of the 26A cytological region of chromosome arm $2 \mathrm{~L}$. Southern blots were made with restriction-cut DNA from overlapping phage clones in the walk. Duplicate blots were probed with ${ }^{32} \mathrm{P}$-labeled cDNA synthesized from adult male or female poly(A) ${ }^{+} \mathrm{RNA}_{\text {; }}$ one restriction fragment from phage $\mathrm{m} 5.5$ was found to hybridize exclusively with the male probe. This 5.2-kb EcoRI restriction fragment was subcloned; a restriction map of this fragment is shown in Figure 1A. Strand-specific RNA probes were generated from this fragment and hybridized to Northern blots of adult male and female poly(A) ${ }^{+}$RNA (Fig. 1B). Two adult male-specific transcripts of approximately $0.9-\mathrm{kb}$ and $0.5 \cdot \mathrm{kb}$ lengths were detected only with the T3 polymerase-generated probe (antisense probe, Fig. 1B, lane 2). A non-sex-specific transcript of $1.6 \mathrm{~kb}$ was also detected occasionally with this probe, and will not be discussed further in this paper (Fig. 1A, dotted line; Fig. 1B, lane 2). The probe from the opposite strand did not hybridize to any detectable transcripts, and no hybridizing bands were detected in poly $(\mathrm{A})^{-}$RNA fractions with either strand-specific probe. The two male-specific transcripts hereafter will be referred to as mst $355 a(0.9 \mathrm{~kb})$ and $m s t$ $355 \mathrm{~b}(0.5 \mathrm{~kb})$, for male-specific transcript, in accordance with the established terminology (Schäfer 1986; DiBenedetto et al. 1987; Chapman and Wolfner 1988). This 5.2$\mathrm{kb}$ genomic fragment contains unique sequences, as only the expected bands were seen when Southern blots of genomic DNA were probed (data not shown).

\section{mst $355 \mathrm{a}$ and mst $355 \mathrm{~b}$ are expressed exclusively in adult male accessory glands}

To determine the tissue(s) in which the male-specific transcripts are expressed, 30 adult Canton $S$ males were dissected into four tissue fractions: (1) accessory gland, (2) testis plus vas, (3) ejaculatory duct plus sperm pump, and (4) the remaining carcass. A Northern blot was made with total RNA extracted from each tissue fraction, and hybridized with the antisense RNA probe. The results are shown in Figure 2A: Both mst 355a and mst 355b are detected exclusively in the accessory gland fraction (lane 1).

To examine the temporal patterns of expression of these transcripts, a Northern blot was made with poly $(\mathrm{A})+$ RNA isolated from both male and female third instar larvae, mid-pupae $(24 \mathrm{hr}$ after puparium formation), and adults (Fig. 2B). Both mst 355a and mst 355b are expressed exclusively in the adult male (lane 6). In a more detailed analysis of the temporal pattern of expression of these transcripts, Chapman and Wolfner (1988)
Figure 1. Map of mst 355 genomic region and transcripts. (A) Restriction map of the mst 355 genomic region. This $5.2-\mathrm{kb}$ EcoRI restriction fragment encodes three transcripts of approximately $1.6 \mathrm{~kb}$ (dotted arrow), and 0.9 and $0.5 \mathrm{~kb}$ (bold arrows); all three transcripts are encoded by the same strand of DNA. The 1.6-kb transcript has not been localized precisely, but it does not overlap with mst $355 a$ or mst $355 b$. Restriction sites are abbreviated: (B) BamHI; (E) EcoRI; (H) HindIII; (R) RsaI; (Xb) XbaI; (Xh) Xhol. (B) Northern blot of $\sim 5 \mu \mathrm{g}$ poly(A) + RNA from adult females and males, probed with a strandspecific RNA probe covering the entire $5.2-\mathrm{kb}$ restriction fragment shown in $A$. The EcoRI restriction fragment was subcloned in the plasmid vector BlueScribe; a ${ }^{32} \mathrm{P}$-labeled antisense RNA probe was generated in vitro by transcription from the $\mathrm{T} 3$ promoter. (Lane 1) Female RNA; (lane 2) male RNA. The antisense probe detects three transcripts: the $1.6-\mathrm{kb}$ transcript is found variably in both male and female adult flies, and the 0.9-kb (mst 355a) and $0.5-\mathrm{kb}$ (mst 355b) transcripts are found only in adult male flies. No transcripts were detected with sense strand RNA probes in either $\operatorname{poly}(\mathrm{A})^{+}$or $\operatorname{poly}(\mathbf{A})^{-}$ fractions (data not shown).

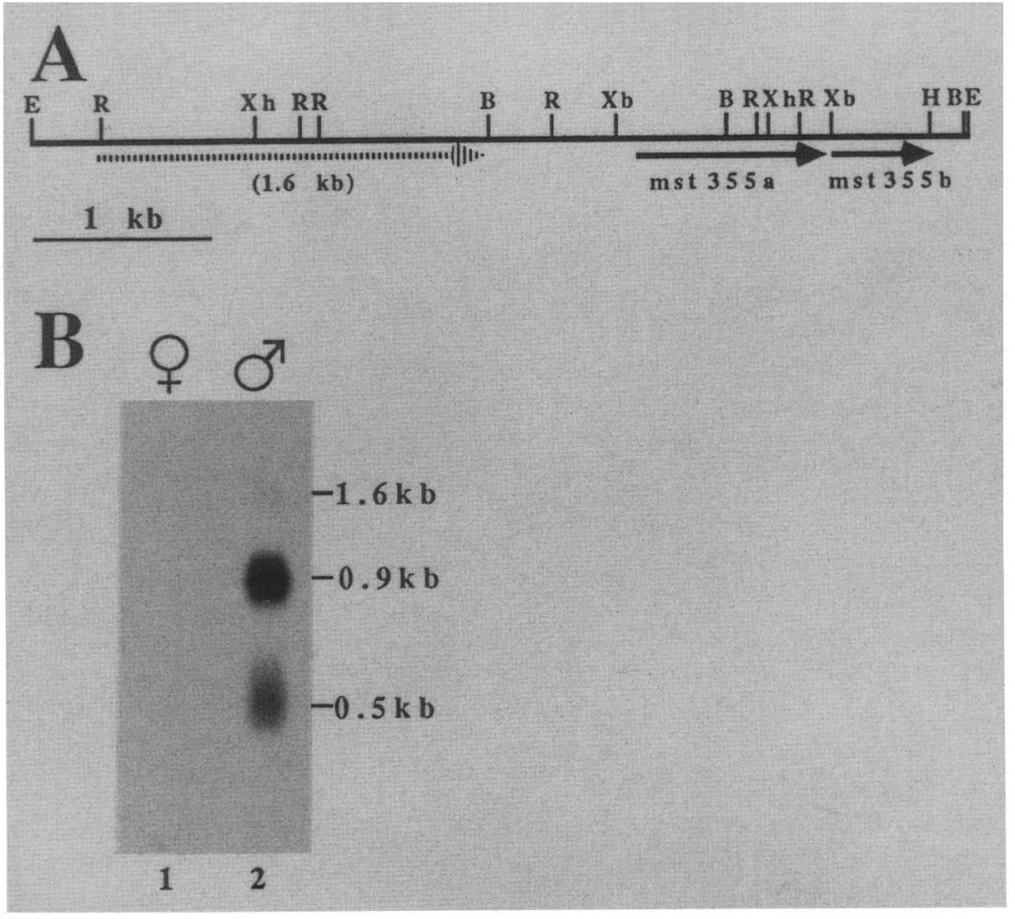




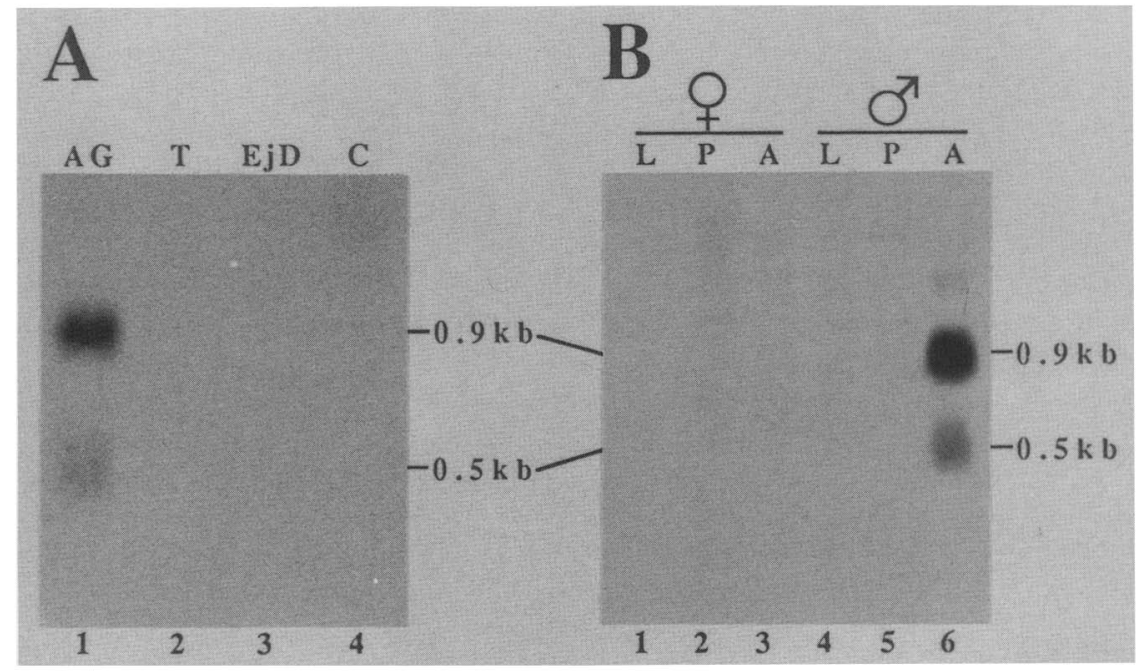

Figure 2. Tissue and temporal patterns of mst expression. (A) Northern blot of total RNA extracted from male tissue fractions. Forty adult male Canton $S$ flies were dissected into four tissue fractions and RNA was extracted. (Lane 1) Accessory gland (AG); (lane 2) testis plus vas $(T)$; (lane 3) ejaculatory duct plus sperm pump (EjD); (lane 4) 10 of the remaining carcasses (C). The probe was a primer-extended genomic DNA fragment containing only mst $355 a$ and mst $355 b$. (B) Northern blot of $\sim 5 \mu \mathrm{g}$ of poly(A) ${ }^{+} \mathrm{RNA}$ extracted from unisex animals (the progeny of cin $y \mathrm{w}$ females) at three developmental stages. (Lanes 1 and 4 ) Third instar larvae (L); (lanes 2 and 5) mid-pupae $24 \mathrm{hr}$ after puparium formation (P); (lanes 3 and 6) 2-day-old adults (A). The probe used was antisense RNA as in Fig. 1B.

have found that mst $355 a$ and mst $355 b$ first become detectable during the late pupal stage P13 (Bainbridge and Bownes 1981), about $12 \mathrm{hr}$ prior to eclosion at $25^{\circ} \mathrm{C}$; maximal levels of RNA accumulation are reached within $24 \mathrm{hr}$ after eclosion.

Expression of mst $355 \mathrm{a}$ and $\mathrm{mst} 355 \mathrm{~b}$ does not require spermatogenesis, and is independent of the state of the tra2 locus in adults

The products of the accessory gland are involved in male fertility, thus the expression of accessory gland products may require normal development of the male germ line. To determine if the expression of mst $355 a$ and mst $355 b$ depends upon spermatogenesis, we examined the adult male progeny of tudor $\left(t u d^{1}\right)$ female flies for the presence or absence of the transcripts. The $t u d^{1}$ mutation is a maternal-effect that blocks the formation of pole cells in all progeny of a $t u d^{1} / t u d^{1}$ mother (Boswell and Mahowald 1985). Since the pole cells generate the germ line of the adult fly, progeny of $t u d^{1}$ mothers lack germ cells and those somatic functions that require the presence of the germ line for their own development. Both mst $355 a$ and mst $355 b$ were found in adult male progeny of $t u d^{1} / t u d^{1}$ mothers (Fig. 3A, lane 2), demonstrating that normal expression of these two transcripts is not dependent upon spermatogenesis.

The male accessory gland is an adult somatic tissue. To see whether the expression of the accessory gland transcripts is affected by genes known to regulate somatic sexual development, we looked for the transcripts in $\mathrm{X} / \mathrm{X}$ flies lacking activity of the $t r a 2^{t s 2}$ gene product. The tra2ts2 gene product is required for normal female somatic development (Belote and Baker 1982). X/X flies lacking tra2ts 2 product throughout development are somatic males, and as adults have accessory glands and other male genital tissues derived from the genital disk. The results (Fig. 3B, lane 5) show that both mst $355 a$ and mst $355 b$ are expressed in such somatically transformed $\mathrm{X} / \mathrm{X}$ adult flies. Restoration of $\mathrm{tra}^{\text {ts } 2}$ activity in adulthood does not abolish the expression of mst $355 a$ or mst $355 b$ (Fig. 3B, lanes 7-10); thus we conclude that these accessory gland genes are no longer regulated by the somatic sex determination gene tra2 $2^{\text {ss } 2}$ at the time of their expression.

Sequence organization of the mst $355 \mathrm{a}$ and $\mathrm{mst} 355 \mathrm{~b}$ genes

The genomic DNA sequence and the organization of the two genes was determined by sequence analysis of genomic and cDNA clones; the results are summarized in a map of the genes (Fig. 4A) and the genomic sequence of the region (Fig. 4B). The $5^{\prime}$ ends of both transcripts were determined by extension of synthetic oligonucleotide primers complementary to the mature mRNAs; one major start site and one minor start site were seen for the upstream transcript (mst 355a), while the downstream transcript (mst 355b) showed a single position for the $5^{\prime}$ end (data not shown; see Methods for details). We have assigned the major start site of mst $355 a$ as +1 ; unless noted otherwise, all positions are indicated relative to this nucleotide. The minor start of mst $355 a$ occurs at position +2 , and the $5^{\prime}$ end of mst $355 b$ occurs at position +1031 .

To isolate cDNA clones of mst $355 a$ and mst $355 b$, we constructed a cDNA library in the vector $\lambda \mathrm{gt} 10$ from poly $(\mathrm{A})^{+}$RNA isolated from adult male flies. From this 


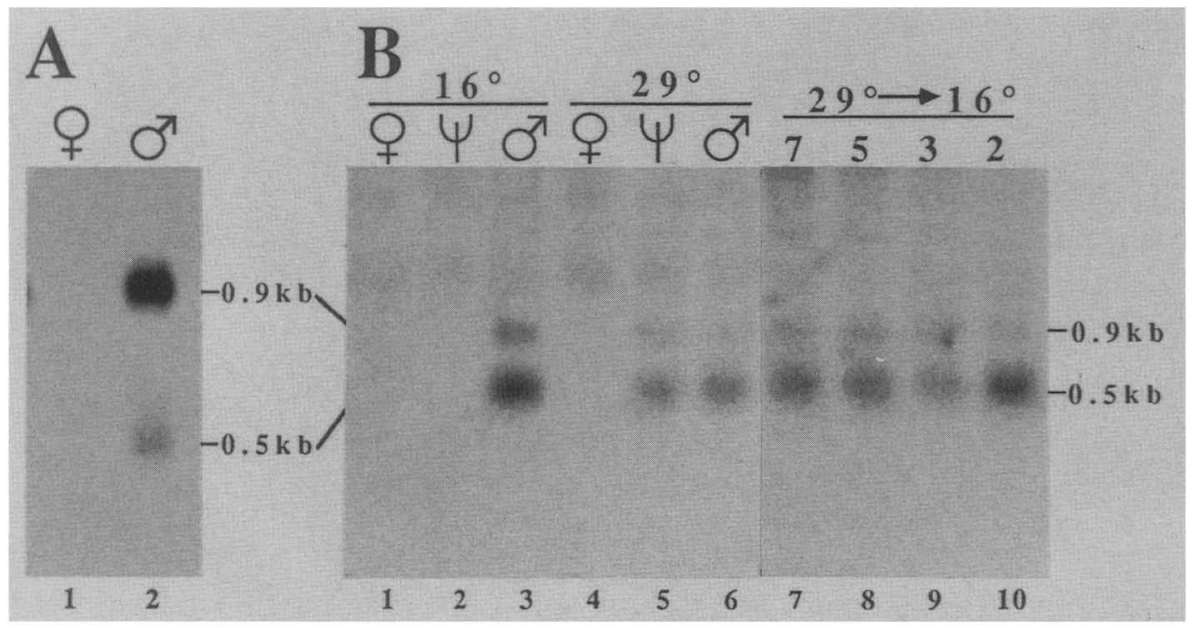

Figure 3. Genetic regulation of mst $355 a$ and $m s t 355 b$ expression. $(A)$ Northern blot of $\sim 5 \mu g$ poly $(A)^{+}$RNA extracted from germ-lineless adult progeny of $b w s p t u d^{1} / b w s p t u d^{1}$ mothers. (Lane 1) Female progeny; (lane 2) male progeny. The probe used was the antisense RNA as in Fig. 1B. (B) Effect of tra2 on mst $355 a$ and mst $355 b$ expression. Northem blot of total RNA extracted from 50 adult flies for each lane. Genotypes of 2-day-old adults, raised and kept at $16^{\circ} \mathrm{C}$ : (lane 1$) \mathrm{X} / \mathrm{X}, \mathrm{tra}^{\mathrm{ts} 2} \mathrm{bw} / \mathrm{CyO} ;($ lane 2$) \mathrm{X} / \mathrm{X}, \mathrm{tra} 2^{\text {ts2 }}$ $b w / t_{r a 2^{t s 2}} b w$; (lane 3) X/Y, $B^{s} ;$ tra2ts2 $b w / t^{t r a 2^{t s 2}} b w$. Genotypes of 2-day-old adults, raised and kept at $29^{\circ} \mathrm{C}:($ lane 4$) \mathrm{X} / \mathrm{X}$, tra2ts2

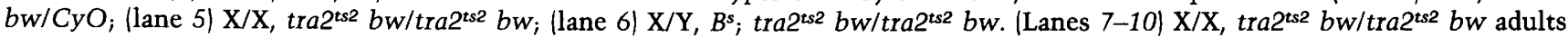
raised to adulthood at $29^{\circ} \mathrm{C}$, then downshifted to $16^{\circ} \mathrm{C}$ for the indicated period of days before RNA extraction. (Lane 7 ) 7 days after downshift; (lane 8) 5 days; (lane 9) 3 days; (lane 10) 2 days. The intensity of $m s t 355 a$ on Northern blots is usually greater than that of mst $355 b$ (see $A$ ), although mst $355 a$ occasionally gives a weaker signal, as on this panel. This may reflect a strain-specific variation.

library we isolated four cDNA clones of mst $355 a$ and two cDNA clones of mst $355 \mathrm{~b}$. All of the cDNA clones were sequenced completely on at least one strand. None of the cDNA clones extended to the 5 ' ends predicted by the primer extension experiments: of the mst $355 a$ clones, one began at nucleotide +20 , two began at nucleotide +151 , and one began at nucleotide +18 , preceded by five bases not found anywhere in the genomic sequence. We searched the genomic sequence for two kilobases upstream of mst $355 a$ for this five-base sequence, but found no matches. Since the fusion point of this pentamer to the genomic sequence bears no resemblance to consensus splice junctions, we regard the extra five bases as an artifact of the cDNA library construction. Of the mst $355 b$ clones, one began at nucleotide +1045 , or nucleotide +16 of $m s t 355 b$; the other cDNA clone began at nucleotide +1050 , or nucleotide +21 of mst $355 \mathrm{~b}$. Except for the unidentified five bases, all of the cDNA sequences matched the genomic sequences exactly. Reasonable matches to established consensus sequences (Mount 1982) were found at each intron boundary.
Two sequence similarities are observed in comparisons of the nucleotide sequences upstream of each transcript. First, at a position 450 bases upstream of mst $355 a$ and 250 bases upstream of mst $355 b$, there is an identity of 18 out of a stretch of 20 nucleotides (small stippled box in Fig. 4A). Second, at a position about 100 bases upstream of both transcripts, there is an identity of 9 out of a stretch of 10 nucleotides (small open box in Fig. 4A). Upstream of mst $355 a$ is a reasonable match to the Goldberg-Hogness consensus sequence, beginning at nucleotide -28: TATATATAA (bold letters in Fig. 4B). Interestingly, upstream of mst $355 b$ there is no match to the Goldberg-Hogness consensus sequence at the appropriate position. The closest match is the polyadenylation signal of mst $355 a$ (TAATAAA), but this begins 48 nucleotides upstream of the first base of $m s t$ $355 b$.

Two of the mst $355 a$ cDNA clones terminate with long poly(A) tracts at +1009 (vertical arrow, Fig. $4 \mathrm{~B}$ ); the other two mst 355a cDNA clones terminate without poly(A) at positions +1002 and +1004 . The two mst $355 b$ cDNA clones are derived from RNAs with distinct

Figure 4. Map and genomic sequence of the mst 355 region. (A) Detailed map mst $355 a$ and mst $355 b$. The boxes under the solid line represent the exons of mst $355 a$ and mst $355 b$. The filled regions of the boxes represent the open reading frames; open regions represent $5^{\prime}$ and $3^{\prime}$ nontranslated sequences. Sites where addition of poly $(A)$ has been found in cDNA clones are indicated by appended $A_{n}$. The small square superimposed on the solid line represent sequence identities; the stippled boxes outline sequence elements having 18 out of 20 identical nucleotides (numbered as in $B$, positions -221 to -202 : GAGGCCAGAA AAATGTATAT and + 541 to +560: GAGGCCAGAA AAACGGATAT); the open boxes outline sequence elements having 9 out of 10 nucleotide sequence identity (positions - 115 to - 106: ATTGCAATAC and +939 to +948: ATTGCAAGAC). Restriction sites are abbreviated: (B) BamHI; (E) EcoRI; (H) HindIII; (R) Rsal; (Xb) XbaI; $(\overline{\mathrm{Xh}})$ XhoI. (B) Genomic sequence of the $\mathrm{mst} 355$ region, from -300 relative to the start site of $m s t 355 a$ to +1736 , the end of the restriction fragment. The TATA element at -28 is shown in bold letters, splice junction sequences are underlined once, and poly(A) addition signals (Birnstiel et al. 1985) are double underlined. The first nucleotide of mst 355b corresponds to nucleotide +1031 of the genomic sequence. Vertical arrows denote poly(A) addition sites, except the last arrow at position +1594 which denotes the $3^{\prime}$ end of one cDNA clone which did not have a poly(A) tail. 


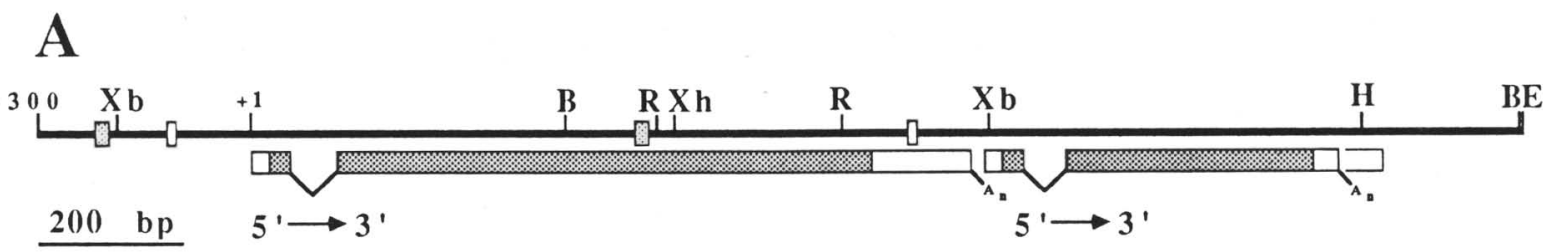

B

-300: AGTCTTAAAATTGTTAGCCTCGACTAAAGCACCCGATGCCAAGAAGAATCTTATAAAAAAAACCGAAGATATCCGTCCGGAGGCCAGAAAAATGTATATA

-200: TCTTAATATCTAGATACTACAGGACTAAAATGTATATTCAACGAATATTTATATATTTTGATGATGTITTTTTTITTTTTGAAAATIGCAATACTACAA

-100: GATCATATTTATAAATATCAAGCAGAACGAAACAAGAGCCGTTGCAAAAGCGCTTATGTAACATGAAATCGIATATATAAGGAATGCAATAATACCTTA

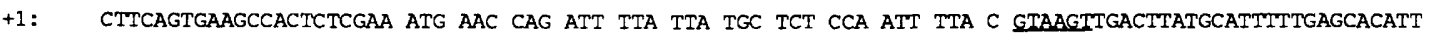
Met Asn Gln Ile Leu Leu Cys Ser Pro Ile Leu L

+90: TAAGAACGAGTATCTTTTCGTTTAG TG CTG CTT TTT ACA GTG GCA AAC TGC GAT AGT GAG CAA CAA CTC GAT TCA GCT ATG

12: eu Leu Leu Phe Thr Val Ala Asn Cys Asp Ser Glu Gin Gin Leu Asp Ser Ala Met

+169: CAC CTG AAA AGT GAT TCT ACG AAA AGT GCA TCT CTG AAA AAT GTT CCT CCC AAG AAT GAT GAG ACA CAG GCC AAA 31: His Leu Lys Ser Asp Ser Thr lys Ser Ala Ser Leu lys Asn Val pro Pro lys Asn Asp Glu Thr Gln Ala Lys

+244: ATA GCC AAA GAT GAT GTA GCT CTG AAA GAT GCG AAA AAG GGC GAT TAT ATA ATG GAT ATC GAT ATT TCT GAT TTG 56: Ile Ala Lys Asp Asp Val Ala leu lys Asp Ala lys lys Gly Asp tyr Ile Met Asp Ile Asp Ile Ser Asp Leu

+319: CCG CTG GAT GAT TAT CCA ATC AAT AGG TCC AAA TCA CTA AAA AGC TCT TCC ATT GAC TTG AAT AAT ATT CCT TTC 81: Pro Leu Asp Asp tyr pro Ile Asn Arg Ser Lys Ser leu Lys Ser Ser Ser Ile Asp Leu Asn Asn Ile Pro phe

+394: AAT AAA GGA CTA GAT GAT TTC CCG GCA AAA GAA AAA AAT CAA GGA TCC AAT CAA AGT GCG CTC AAG GCC CTG CAA 106: Asn lys Gly Leu Asp Asp phe Pro Ala lys Glu Lys Asn Gln Gly Ser Asn Gln Ser Ala leu Lys Ala Leu Gln

+469: CAG AGg tTA CTA ACG GAg CAG AAC AAT AGT TTA CTT CTC CGg AAC CAT TCC ATA TAC TTG ATG AAA GAA ATA GAG 131: Gln Arg leu Leu Thr glu Gln Asn Asn Ser leu Leu leu Arg Asn H1s Ser Ile Tyr Leu Met Lys Glu Ile Glu

+544: GCC AGA AAA ACG GAT ATT ATC AAA GTA CGA CAG TTA AAC CTC GAT TTA GAG CTC GAG CTA AAT ACT GTG AAC CGC 156: Ala Arg Lys Thr Asp Ile Ile Lys Val Arg Gln Leu Asn Leu Asp leu Glu Leu Glu Leu Asn Thr Val Asn Arg

+619: AGA CTT TTG GAA TTG AAT GGG CAA CTG CAA AAC ACT CGA AAG ICC ACA AAG CCG TGT AAG AAA CGT TCT AGT AAG 181: Arg Leu leu Glu Leu Asn Gly Gin leu Gln Asn Thr Arg lys Ser Thr lys pro Cys Lys lys Arg Ser Ser lys

+694: GAI AGC GCC CCA CCT GCC GCC AAT CAG TTT CAG GAA GCC AAC GTC AGG AAC ACT TAC CGT AAC AAA TAT CTA ACA 206: Asp Ser Ala Pro pro Ala Ala Asn Gln phe Gln Glu Ala Asn Val Arg Asn thr Tyr Arg Asn Lys Tyr Leu Thr

+769: CTT CTG AAA GAA CTT AGT CAG AAG ATC AAT AAC GAA ATC GCG AAA GTC GCT ACC GAT GTA CCC ACG GAG ACA AAT 231: Leu Leu Lys Glu Leu Ser Gln Lys Ile Asn Asn Glu Ile Ala Lys Val Ala thr Asp Val Pro Thr Glu Thr Asn

+844: CCT TCC CAA GGG AAT CTA CCA ACA CIT TAA TAG TTAAAATCATATGGTTTCCCATCATTTAAGAACAGAAACAATAGATTGAGTCAT 256: Pro Ser GIn Gly Asn Leu Pro Thr Leu END END

+931: TGCAAGACCTTCTAATTGCGCACTTACTTATTTTTCTGATCTTGTTAGCCGTAATAAATGCGATCATAACGCTTAGGCCAAGTGCTGGTTGGATTCTTI A

+1031: ACAGTCTAGAGAATACGTTTCCACA ATG AAC TAC TTC GCG GTG ATC TGC ATT TTC I GTAAGTTCGTAAGGATCTTCGAGGTTCTTAGA $+1$ Met Asn Tyr phe Ala Val Ile Cys Ile phe $s$

+1118: GTTTGCCTAATTTAGTTTTCATCACTTAG CC TGC ATT TGC CTT TGG CAA TTT AGC GAT GCT GCC CCC TTT ATA AGC GTT CAG $11:$ er Cys Ile Cys Leu Trp Gln Phe Ser Asp Ala Ala Pro Phe Ile Ser Val Gin

+1200: TCT AGT TCA CAA TCA AGA TCC CAG AAA GTG ATG AAT GGC ATG TTG AGA ACC CTA TAC GAC TAC AGT GTT CAG GAT 28: Ser Ser Ser Gin Ser Arg Ser Gln Lys Val Met Asn Gly Met Leu Arg Thr Leu Tyr Asp Tyr Ser Val Gin Asp

+1275: AGT GTG AAC GAT GCG ACT GGG CAT TTG ATA CAA ACT CAC AAA GCA GAC TTC AAC TCG GAT GTC ATG AGT CCT GAT 53: Ser Val Asn Asp Ala Thr Gly H1s Leu Ile Gln Thr His Lys Ala Asp Phe Asn Ser Asp Val Met Ser Pro Asp

+1350: GAA ATA GAG AGT GTG CGC CAG CAA CTG AAC ATG GCA TAA TTTTGGATTCACCAGCAAATATCTTAAACAGCGATTATTGCATATGC 78: Glu Ile Glu Ser Val Arg Gln Gln Leu Asn Met Ala END

+1436: TTTTTAACCGCAGTTACTTCAAGCAAAATATTATGTATCTATGCAATATGTAATATATCATTTAAAAGATATAATAACCCAAATAAAGTATTTACCATA

+1536: TTATAATGGACATGGACTCTAAGAAGCTTTTGAAATAAGAGTTCCGATATTTCCAAAGCTGTGATAAAATTGTATTGGAAATATAGAGTGGGTATATGG A

+1636: TATTTGTAGTTATACATATAAAATGTTATACATATAAAATGCAGACGAAAGGACATAAATATAAAATACATTTTATGACATAAACTCGACCTGTGGGTC

+1736: ATAAAGATTCGTCTAGCTCAACTTTCACAAATATGGGATCCGGGGAATC

Figure 4. (See facing page for legend.) 
$3^{\prime}$ ends (vertical arrows, Fig. 4B): one contains 19 A residues beginning at nucleotide +1539 while the longer clone terminates at nucleotide +1594 without a poly(A) tract. The significance of this $3^{\prime}$ heterogeneity is unknown, as the only long open reading frame of mst $355 b$ terminates before the first polyadenylation site. Our Northern blots show a diffuse band for mst $355 b$, probably due to variability in the length of the poly(A) tails; thus the relative contribution from species utilizing either poly|A| site cannot be distinguished. The predicted sizes of the mature transcripts, excluding the poly|A) tail, are 957 nucleotides for mst 355a, and 448 and 504 nucleotides for mst $355 b$.

Finally, each transcript contains only one long $1>50$ amino acid) open reading frame beginning with ATG. The ORF of mst $355 a$ encodes a basic protein of 264 amino acids, hereafter called msP $355 a$. The ORF of mst $355 b$ encodes an acidic protein of 90 amino acids, hereafter termed $m s \boldsymbol{P} 355 b$.
Both msP 355a and msP 355b have features of secreted proteins

As the male accessory gland is a secretory tissue, proteins encoded by accessory gland RNAs would be expected to have hydrophobic amino-terminal signal sequences (Harwood 1980). As seen in hydrophilicity profiles of the predicted amino acid sequences (Fig. 5A,B), both $m s \boldsymbol{P} 355 a$ and $m s \boldsymbol{P} 355 b$ exhibit hydrophobic amino termini. Examination of these hydrophobic segments for potential signal peptide cleavage sites using the methods of von Heijne (1983) predicts positions with a high probability of cleavage, after amino acid 21 of the predicted $m s \boldsymbol{P} 355 a$, and after amino acid 18 of the predicted $m s \boldsymbol{P} 355 b$ (arrows, Fig. 5C). Thus, both msP $355 a$ and $m s \boldsymbol{P} 355 b$ are potentially secreted proteins.

We searched the amino acid sequence of $m s \boldsymbol{P} 355 a$ for other signals commonly found on secreted proteins. The sequence Asn-X-Ser/Thr is a signal for $N$-linked
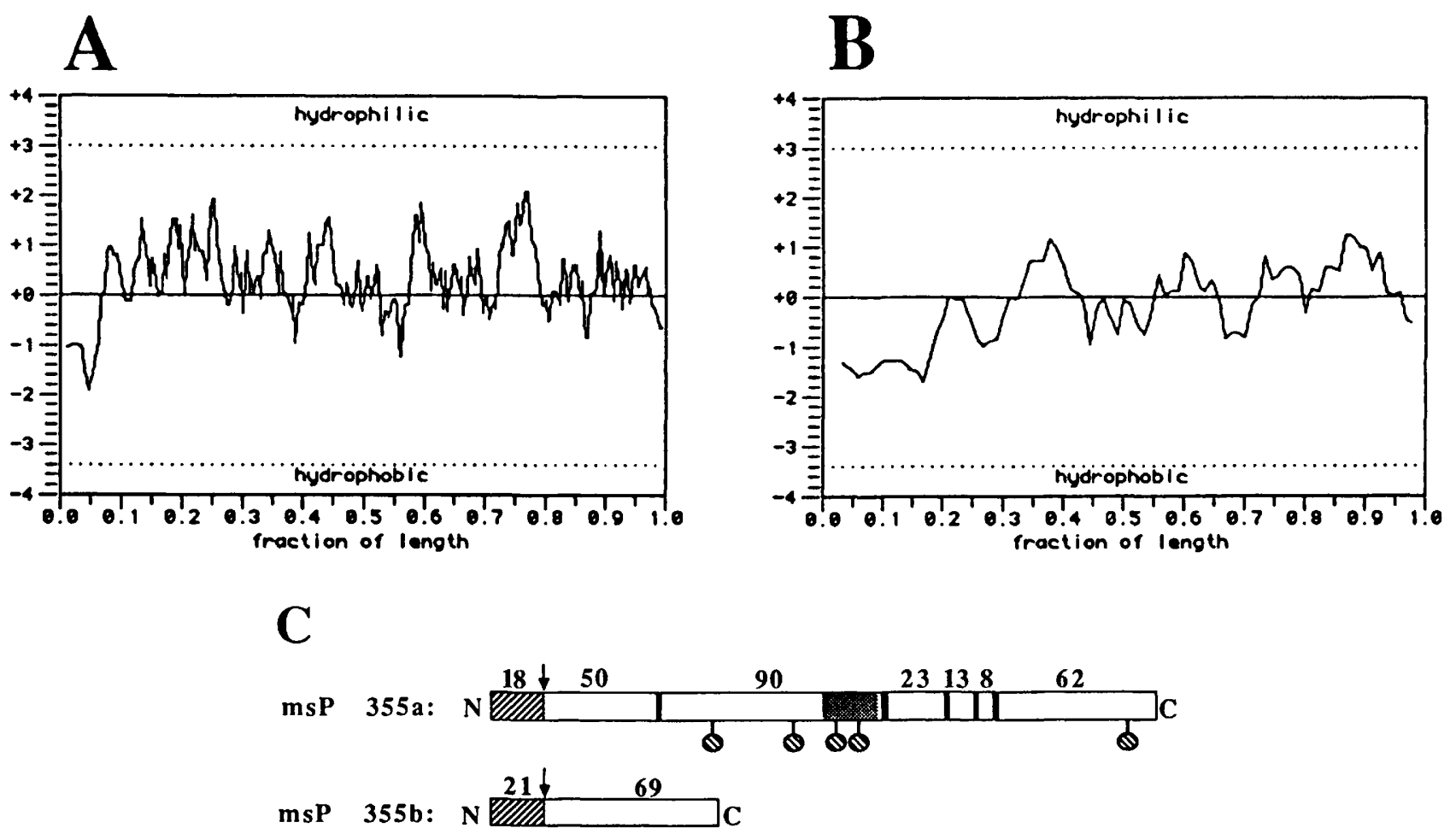

Figure 5. Features of the predicted proteins. $(A)$ Hopp-Woods hydrophilicity profile of the predicted $\mathrm{ms} \boldsymbol{P} 355 \mathrm{a}$ amino acid sequence. This profile was determined with an averaging length of 6 amino acids (Hopp and Woods 1981), using the DNA Inspector II + sequence analysis program (Gross 1986). The abscissa represents fractional length of the protein, which is a total of 264 amino acids long. $(B)$ Hopp-Woods hydrophilicity profile of the predicted $m s \boldsymbol{P} 355 b$ amino acid sequence. This profile was determined as in $A$; the length of this protein is 90 amino acids. $|C|$ Maps of $m s P 355 a$ and $m s P 355$. Hatched segments represent hydrophobic stretches at the amino terminus of each sequence. Vertical arrows represent signal peptide cleavage sites predicted by the methods of von Heijne (1983). Small circles below msP 355a mark $N$-linked glycosylation signals (Asn-X-Ser/Thr; Kornfeld and Kornfeld 1985). Small solid bars represent possible peptidase cleavage sites. The stippled area represents region of identity between $\mathrm{ms} \boldsymbol{P} 355 a$ and ELH (aligned sequences are shown in Fig. 7). Small numbers above each diagram indicate distance between adjacent cleavage sites in amino acids. The predicted $m s \boldsymbol{P} 355 a$ would have a net charge of +5 at $\mathrm{pH} 7.0$, and the predicted $m s \boldsymbol{P} 355 b$ would have a net charge of -4 at pH 7.0 . 
glycosylation of secretory proteins (Kornfeld and Kornfeld 1985). The predicted $m s \boldsymbol{P} 355 a$ sequence contains five such $N$-linked glycosylation signals, four of which are clustered near the central region of the protein, and the fifth of which lies near the carboxyl terminus /circles in Fig. $5 \mathrm{C}$ ). No such signals are found in the predicted sequence of $m s \boldsymbol{P} 355 b$.

Peptide hormones and peptide pheromones, such as yeast mating factor (Kurjan and Herskowitz 1982), usually are synthesized in the form of larger precursor proteins, which are later cleaved at specific positions to release the bioactive peptides. Pairs of basic amino acids (Lys or Arg) are frequently the sites of such specific peptidase cleavages (Giesow and Smyth 1980). The predicted $m s \boldsymbol{P} 355 a$ sequence contains five such pairs of basic amino acids, at amino acid positions $67,157,180$, 194, and 201 (solid crossbars in Fig. 5C). Thus, msP $355 a$ encodes a secreted protein (see below) which may also be glycosylated and cleaved into smaller peptides, in the manner of known precursors to peptide pheromones and hormones.

\section{msP 355a is secreted and transferred to the female during mating}

To follow the protein encoded by mst 355a, antibodies against a $\beta$-galactosidase fusion protein were raised in rabbits. The antiserum was used to probe Western blots of proteins extracted from various Drosophila tissues. Extracts were prepared from male accessory glands and from the internal genitalia (excluding the ovaries) of unmated (virgin) female flies. Anti-msP 355 a recognizes three male accessory gland-specific bands of $M_{r} 41,37$, and $36 \mathrm{kD}$ (Fig. 6, lane 1), of which the $37-\mathrm{kD}$ band is the

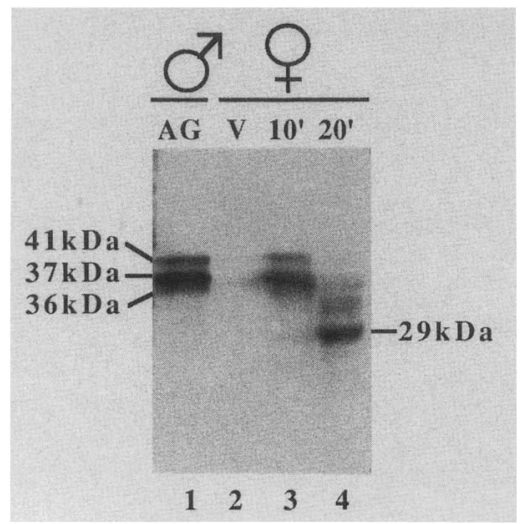

Figure 6. Immunological detection of $m s \boldsymbol{P} 355 a$. Western blot probed with anti-msP $355 a$. Proteins were extracted from dissected tissues of 10 flies and run on $12.5 \%$ polyacrylamideSDS gels. (Lane 1) Male accessory glands; (lane 2) internal genital ducts (excluding ovaries) of virgin female flies; (lane 3) internal genital ducts (excluding ovaries) of mated female flies, dissected $10 \mathrm{~min}$ after the beginning of copulation; (lane 4) internal genital ducts (excluding ovaries) of mated female flies, dissected at end of mating, $20 \mathrm{~min}$ after beginning of copulation. The three accessory gland bands detected with anti-msP $355 a$ at $41 \mathrm{kD}, 37 \mathrm{kD}$, and $36 \mathrm{kD}$ are indicated; in lane 4 the main band has shifted down and now migrates at $29 \mathrm{kD}$. most intensely stained. These bands are not present in the unmated female flies (Fig. 6, lane 2), and they migrate slower than would be expected from the amino acid sequence alone, which predicts an $M_{r}$ of $29 \mathrm{kD}$ (including the putative signal peptide). We consider these three bands to be alternate forms of the protein encoded by $m s t 355 a$, and in this paper we will refer to the set collectively as $m s \boldsymbol{P} 355 a$.

During Drosophila mating, ejaculation normally occurs between 5-10 min after the beginning of copulation, which normally lasts for about 20 min (Fowler 1973). Mating couples were interrupted either after 10 min of copulation or allowed to complete copulation, and protein extracts were prepared from the internal genitalia (excluding ovaries) of the females. $m s \boldsymbol{P} 355 a$ is transferred to the female fly during the first $10 \mathrm{~min}$ of copulation (Fig. 6, lane 3). At the end of mating, 10-15 min after ejaculation, $m s \boldsymbol{P} 355 a$ has been altered; the major band now appears at $29 \mathrm{kD}$ (Fig. 6, lane 4).

Preliminary results using antibodies raised against an $m s \boldsymbol{P} 355 b-1 a c Z$ fusion protein indicate that $m s \boldsymbol{P} 355 b$ also encodes an accessory gland protein that is transferred to the female genital tract during mating. The accessory gland protein which is recognized by anti-ms $\boldsymbol{P}$ $355 b$ migrates at $11-14 \mathrm{kD}$, which is close to the $10-\mathrm{kD}$ size (including the putative signal peptide) predicted from the sequence of $m s \boldsymbol{P} 355 b$.

msP 355a has amino acid sequence similarity to Aplysia egg-laying hormone

The National Biomedical Research Foundation's protein sequence database was searched for any matches to the predicted $m s \boldsymbol{P} 355 a$ and $m s \boldsymbol{P} 355 b$ amino acid sequences, using MicroGenie software (Beckman). No significant sequence matches were found for $m s \boldsymbol{P} 355 b$. One match was found for the msP $355 a$ amino acid sequence, with egg-laying hormone (ELH) of the mollusc Aplysia californica (the California seahare). ELH is a 36amino-acid peptide neurohormone which is involved in eliciting stereotyped postmating behavior in Aplysia (Chiu et al. 1979). The similarity lies in the stretch from amino acids 121 to 137 of $m s \boldsymbol{P} 355 a$, in which 11 out of 17 amino acids are identical to those in the stretch from amino acid 2 to 18 of ELH, allowing for a single amino acid gap in each sequence (Fig. 7).

\section{Discussion}

The male accessory gland of Drosophila produces a complex secretion of amino acids, proteins, peptides, lipids, and carbohydrates (Federer and Chen 1982; Chen 1984). Certain components of the accessory gland secretion elicit specific behavioral and physiological responses in the female fly after mating (Chen 1984); others have been suggested to play a role in sperm storage and nutrition (Fowler 1973). While the accessory gland secretion of $D$. melanogaster has been shown to increase oviposition and decrease receptivity, little progress has been made in the analysis of specific secretion 


\section{Aplysia ELH: 1 Ile Ser Ile Asn GIn Asp Leu Lys Ala Ile Thr Asp Met Leu Leu Thr Glu GIn msP 355a: ...Gly Ser 120 \\ Ile Arg Glu Arg Gin Arg Tyr Leu Ala Asp Leu Arg Gin Arg Leu Leu Glu Lys Asn Asn Ser Leu Leu Leu Arg Asn His Ser lle Tyr Leu Met Lys Glu Ile Glu.. 155}

Figure 7. Amino acid alignment of $m s \boldsymbol{P} 355 a$ with ELH. The regions of $m s \boldsymbol{P} 355 a$ sequence identity with ELH are outlined; the full sequence of ELH (Chiu et al. 1979) is presented for clarity. Allowing one gap in each sequence, 11 of 17 amino acids are identical (boxed); if no gaps are allowed, 9 of 17 amino acids are identical. Amino acid 155 of $m s \boldsymbol{P} 355 a$ is followed by the possible cleavage signal Arg-Lys; amino acid 36 of ELH is followed by Gly-Lys-Arg. The probability of finding 9 identities in a stretch of 17 amino acids is $3.1 \times 10^{-6}$ as calculated by the method of Fitch (1966), using the amino acid frequencies in the ELH precursor (271 amino acids; Nambu and Scheller 1986) and $m s \boldsymbol{P} 355 a$ (264 amino acids). We feel that this match has biological significance, because the probability of such a random match is so low, and because there are only $6.3 \times 10^{4}$ possible 17-amino-acid comparisons possible between the ELH precursor and $m s P$ 355a (Fitch 1966).

components. Two components of the $D$. funebris secretion have been purified and linked to specific aspects of the female response to mating (Baumann 1974b); however, the $D$. funebris components do not function in $D$. melanogaster (Baumann 1974b). We have taken a molecular genetic approach to analyze the functions of accessory gland products by examining the structure and expression of genes that encode components of the accessory gland secretion. This information will allow a more detailed analysis of the roles of the protein products of these genes in reproduction.

\section{Expression of mst $355 \mathrm{a}$ and $\mathrm{mst} 355 \mathrm{~b}$}

We report here that the accumulation of mst $355 a$ and mst $355 b$ RNAs is not controlled by the somatic sex determination hierarchy in adults. A similar pattern of regulation is observed for another male accessory gland specific transcript, mst 316 (DiBenedetto et al. 1987). Chapman and Wolfner (1988) have shown that adult expression of mst $355 a$, mst $355 b$, and mst 316 is specified by the sex determination loci during the late third larval instar. At this time, some of the cells of the genital imaginal disk become determined to produce accessory glands (Chapman and Wolfner 1988). During the third larval instar, alteration of the expression of $t r a 2^{t s 2}$ by temperature shift can affect subsequent adult expression of mst $355 a$ and mst $355 \mathrm{~b}$. As shown here, alteration of tra2 ${ }^{\text {ts } 2}$ expression in the adult can no longer affect adult expression of mst $355 a$ and $m s t 355 b$. We have also shown that expression of the male accessory gland transcripts mst $355 a$ and mst $355 b$ does not depend upon a functional germ line. Male progeny of $t u d^{1}$ mothers, without germ lines, express both mst 355a and mst $355 b$ at essentially wild-type levels, and these animals have normal appearing accessory glands.

Taken together, these analyses of the expression of mst $355 a$ and mst $355 b$ suggest that the regulation of these genes by the somatic sex determination genes (such as tra2) occurs at the level of tissue specificity. The somatic sex determination hierarchy establishes whether any male accessory gland tissue will develop from the genital disk; as a consequence of accessory gland differentiation, both mst $355 a$ and mst $355 b$ tran- scripts are expressed. Once the genital disk cells have become determined to produce accessory glands, alteration of tra2 expression does not affect mst 355a or mst $355 b$ expression (at the level of RNA accumulation).

\section{Gene structure}

The organization of the genes encoding mst $355 a$ and $m s t 355 b$ is unusual, in that the first nucleotide of mst $355 b$ occurs only 20 nucleotides downstream of the mst $355 a$ polyadenylation site. Although it is formally possible that both transcripts are alternate processing products from a single RNA precursor, we have detected no such precursor in primer extension or S1 nuclease experiments. Other preliminary evidence indicates that mst $355 a$ and $m s t 355 b$ are the products of independent transcription units (S.A. Monsma and M.F. Wolfner, in prep.). An mst 355b-lacZ fusion gene which lacks all sequences $5^{\prime}$ to the intron of mst $355 a$ is transcribed with the same sex and tissue specificity as the endogenous mst $355 b$ gene. Therefore, transcription of mst $355 b$ requires neither sequences upstream of mst $355 a$ nor the initiation site $(+1)$ of mst $355 a$.

The mst 355a gene has a good TATA homology at -28 , a typical position. In contrast, the only TATA homology upstream of mst $355 b$ is the polyadenylation signal of mst $355 a$, which, at -48 relative to the start of mst $355 b$, lies further upstream than is typical for this type of regulatory element (Breathnach and Chambon 1981). Thus, the mst $355 b$ transcription unit may not have a functional TATA element. This is not uncommon in Drosophila, as several other Drosophila genes lacking TATA homologies have been described (e.g., Mlodzik and Gehring 1987; Scholnick et al. 1987; Bowtell et al. 1988; Reinke et al. 1988; Sun et al. 1988).

\section{Towards the function of $\mathrm{msP} 355 \mathrm{a}$ and $\mathrm{msP} 355 \mathrm{~b}$}

The male accessory gland produces secreted products that play a role in the postmating response of female flies. We have shown that the antiserum raised against the protein encoded by mst $355 a$ recognizes a set of accessory gland protein bands, referred to as $m s \boldsymbol{P} 355 a$. We have also demonstrated transfer of $m s \boldsymbol{P} 355 a$ from the 
male to the female fly during copulation, and have shown that modification of msP $355 a$ occurs in the female genital tract within 10-15 min after ejaculation. Transfer of msP $355 a$ to the female fly may imply a role in the female postmating response.

The protein encoded by mst $355 a$ has many features associated with peptide pheromone and hormone precursors. Aside from the putative signal peptide, the sequence of $m s \boldsymbol{P} 355 a$ also exhibits five $N$-linked glycosylation signals. Modification of $m s \boldsymbol{P} 355 a$ by addition of carbohydrate does occur, and accounts for some of the difference between the observed $M_{r}$ of $36-41 \mathrm{kD}$ and the predicted $M_{r}$ of $29 \mathrm{kD}$ (S.A. Monsma, unpubl.). msP $355 a$ also contains five pairs of basic amino acids, commonly used as cleavage signals in polyprotein precursors. The observed shift in msP $355 a$ mobility by the end of mating may reflect proteolytic or glycolytic cleavage in the female genital tract. Such cleavage might serve to liberate physiologically active components from $\mathrm{ms} \boldsymbol{P}$ $355 a$.

The predicted amino acid sequence of $\mathrm{ms} \boldsymbol{P} 355 a$ has no similarity to the reported amino acid sequence of the $D$. funebris peptide PS-1, which affects the receptivity of female D. funebris (Baumann et al. 1975). However, $m s \boldsymbol{P}$ $355 a$ has an intriguing characteristic-its region of amino acid sequence similarity with ELH of $A$. californica. ELH is a 36-amino acid peptide hormone that is released from the bag cells of the abdominal ganglion as a result of mating (Kaldany et al. 1985). ELH stimulates specific neurons of the abdominal, pedal, and buccal ganglia, thus contributing to the production of several stereotyped egg-laying behavior patterns (Scheller et al. 1983). ELH also circulates in the bloodstream and stimulates contraction of the ovotestis, contributing to release of the egg mass. Thus, ELH functions both as a neurotransmitter and as a peptide hormone. ELH is contained within a 271-amino-acid precursor protein (Nambu and Scheller 1986), and is released from this precursor by cleavage at paired basic amino acids. In this light, it is intriguing that $m s \boldsymbol{P} 355 a$ contains a pair of basic amino acids at the same position, relative to the region of ELH identity, as the authentic cleavage site at the carboxyl terminus of ELH (see Fig. 7 legend). Cleavage of $m s P$ $355 a$ at this site and at the nearest pair of basic amino acids to the amino terminus would result in a 90-aminoacid peptide, with the similarity to ELH occurring between amino acids 65 and 80 of the ms $\boldsymbol{P} 355 a$ peptide.

One other Drosophila protein, the product of the dunce gene $\left(d n c^{+}\right)$, has been found to have sequence similarity to the ELH precursor (Chen et al. 1986). However, the region of ELH with similarity to the $d n c^{+}$ product is distinct from the region with similarity to $m s \boldsymbol{P} 355 a$. In addition to the learning disability of $d n c$ mutants, Chen et al. note that $d n c$ females are sterile, partly due to their failure to lay eggs. This female sterility phenotype may be genetically suppressed independently of the learning defect, suggesting that the $d n n c^{+}$ product may have independent functions in both learning and in fertility.

At present, the amino acid sequence similarity be- tween $m s \boldsymbol{P} 355 a$ and ELH allows only speculation as to the function of $m s \boldsymbol{P} 355 a$. Both ELH and the secretions of the accessory gland are involved in the production of post mating behavioral and physiological responses. If the amino acid sequence similarity we observed reflects a functional relationship, it would suggest that $m s \boldsymbol{P}$ $355 a$ or its cleavage products might be involved in the increased egg-production rate of the mated female's ovary, or in eliciting oviposition behavior. These hypotheses can be tested by application of $m s \boldsymbol{P} 355 a$ to isolated ovaries or by injection into virgin female flies, and by using the antibodies to follow msP $355 a$ in mated female flies.

\section{Methods}

Fly handling and rearing

Drosophila were raised on yeast-glucose medium at $25^{\circ} \mathrm{C}$ unless otherwise noted. For preparation of RNA or protein, flies were collected within $8 \mathrm{hr}$ of eclosion and aged for 2 days at $25^{\circ} \mathrm{C}$ in the absence of the opposite sex. All protein experiments were carried out with Canton S flies; unless noted otherwise, RNA experiments were carried out with Canton $S$ flies or with unisex cultures derived from the cross of $\operatorname{cin} y w$ as described in DiBenedetto et al. (1987). Where tested, the same results were obtained with Canton $\mathrm{S}$ as with cin progeny.

For experiments to determine the genetic regulation of the accessory gland transcripts, $\mathrm{X} / \mathrm{X}$ tra ${ }^{\text {ts } 2}$ homozygotes were obtained from the $B^{s} Y$; tra ${ }^{t s 2} b w / C y O$ stock, and used as in DiBenedetto et al. (1987). Flies with no germ line were generated by crossing $b w$ sp tud ${ }^{1}$ virgin females to Canton $S$ males as described in DiBenedetto et al. (1987).

Tissues for RNA or protein preparation were obtained by dissection in $0.7 \% \mathrm{wt} / \mathrm{vol} \mathrm{NaCl}$, and were extracted immediately as described below. Fly matings for protein experiments were performed by adding unanesthetized 2-day-old virgin female Canton $\mathrm{S}$ flies to vials containing excess unanesthetized 2-dayold virgin male Canton S flies. Couples were removed to empty vials within $5 \mathrm{~min}$ of the beginning of copulation. Couples were either anesthetized with $\mathrm{CO}_{2}$ at $10 \mathrm{~min}$ after the beginning of copulation and vortexed to separate the flies, or allowed to complete copulation and anesthetized at $20 \mathrm{~min}$ after the beginning of mating. The internal genitalia (excluding the ovaries) of 10 mated female flies from each time point were dissected immediately and extracted as described below.

\section{Nucleic Acids}

Cloning and routine nucleic acid manipulations (Southern blots, plaque filter screening) were performed as described in Maniatis et al. (1982). RNA extractions and Northern blots were performed as in DiBenedetto et al. (1987). Nested deletions of the genomic subclones for sequencing were obtained using exonuclease III (Henikoff 1984) and mung bean nuclease (Komalski et al. 1976). Sequencing was performed by the dideoxy chain-termination method (Sanger et al. 1977), with the addition of deoxy-7-deaza-GTP (Mizusawa et al. 1986) to resolve compression artifacts. Oligonucleotide primers were synthesized by the Cornell Biotechnology Facility. The primer synthesized for mst 355a was complementary to exonic sequences to either side of the intron, and had the sequence 5'-AAAAGCAGCAGTAAAATTGG-3'. The primer synthesized for $m s t$ $355 b$ was complementary to positions +1192 to +1211 of the 
genomic sequence (Fig. 4) and had the sequence 5'TTGTGAACTAGACTGAACGC-3'.

The cDNA library was constructed from $5 \mu \mathrm{g}$ of poly $(\mathrm{A})^{+}$ RNA extracted from 2-day-old adult male progeny of the cin $y$ $w$ cross described in DiBenedetto et al. (1987). Double-stranded cDNA was synthesized and inserted into $\lambda$ gt 10 arms using commercial kits and protocols (Amersham). Size-fractionation steps were omitted, except for removal of excess linkers on a gel filtration column. Independent recombinant phage $2.2 \times 10^{5}$ were obtained; a total of $1 \times 10^{5}$ phage were screened to obtain cDNA clones.

\section{Production of fusion proteins and immunological techniques}

The lacZ-msP 335a fusion was constructed using the pWR5901 bacterial expression vector (Guo et al. 1984). This vector contains the lac regulatory sequences and about 600 amino acids of the amino terminal of $l a c Z$, followed by a multiple cloning site into which a cDNA clone of mst $355 a$ was inserted. Fusion protein expression was induced in Escherichia coli strain JM101 by addition of $3 \mathrm{mM}$ IPTG to the culture medium; a fusion protein of $94 \mathrm{kD}$ was purified from $10 \%$ acrylamide-SDS gels by electroelution from gel slices. One hundred micrograms of the fusion protein was emulsified with Freund's complete adjuvant (Sigma), and injected into giant chinchilla rabbits at multiple subdermal sites. Two weeks after the initial immunization, the rabbits were boosted with $50 \mu \mathrm{g}$ of fusion protein emulsified with Freund's incomplete adjuvant (Sigma). Beginning 2 weeks after the boost, the rabbits were bled twice weekly and boosted as necessary.

Drosophila protein extracts were prepared by grinding whole flies or dissected tissues in lysis buffer [1.5\% SDS, $2.5 \% \beta$-mercaptoethanol, $25 \mathrm{mM}$ Tris- $\mathrm{HCl}(\mathrm{pH} 7.5), 0.05 \%$ bromphenol blue, $5 \%$ glycerol] and heating at $100^{\circ} \mathrm{C}$ for $5 \mathrm{~min}$. Just prior to electrophoresis, the samples were spun in a microfuge for 1 $\mathrm{min}$, and the supernatants reheated briefly $\left(100^{\circ} \mathrm{C}\right.$ for $\left.1 \mathrm{~min}\right)$. SDS gels were $12.5 \%$ polyacrylamide $(36.5$ parts acrylamide : 1.0 part bis-acrylamide; Laemmli 1970). Electrophoretic transfer to nitrocellulose was performed as described in Towbin et al. (1979), with the addition of $0.1 \%$ SDS to the transfer buffer. Western blots were blocked by incubation in TBS $\$ 500$ $\mathrm{mm} \mathrm{NaCl}, 20 \mathrm{~mm}$ Tris- $\mathrm{HCl}, \mathrm{pH} 7.5$ ) containing $3 \%$ gelatin for $45 \mathrm{~min}$. The blots were incubated for $3-16 \mathrm{hr}$ with rabbit antiserum diluted $1: 500$ in TBS containing $1 \%$ gelatin and $0.5 \%$ Tween 20 . The blots were washed $3 \times$ for $5 \mathrm{~min}$ in TBS with $0.5 \%$ Tween-20, then incubated for $1 \mathrm{hr}$ with alkaline phosphatase conjugated goat anti-rabbit IgG (Sigma) diluted $1: 3000$ in TBS containing $1 \%$ gelatin. After washing $2 \times$ for $5 \mathrm{~min}$ in TBS with $0.5 \%$ Tween- 20 and once for $5 \mathrm{~min}$ in TBS, color development was carried out with 5-bromo-4-chloro-3-indolyl-phosphate (Sigma) and nitro blue tetrazolium (Sigma).

\section{Note}

Sequence data described in this paper have been submitted to the EMBL/GenBank Data Libraries under accession number Y00219.

\section{Acknowledgments}

Many thanks to Kevin W. Kraus for stimulating discussion, Ross MacIntyre for the gift of phage m5.5 and the statistical analysis of the similarity between ELH and $m s \boldsymbol{P} 355 a, \mathrm{~K}$. Louise Hanson and Angela DiBenedetto for the identification of phage m5.5, and Heidi Harada for the preparation of Northern blots. Thanks also to Kevin W. Kraus, John T. Lis, and Michael L. Goldberg for comments on the manuscript. This research was supported by National Institutes of Health grant GM33030 and American Cancer Society grant NP590 to M.F.W., and by a National Science Foundation Graduate Fellowship and a National Institutes of Health Training Grant 5 t32 GM07273 to S.A.M.

\section{References}

Bainbridge, S.P. and M. Bownes. 1981. Staging the metamorphosis of Drosophila melanogaster. I. Embryol. Exp. Morphol. 66: 57-80.

Baumann, H. 1974a. The isolation, partial characterization, and biosynthesis of the paragonial substances, PS-1 and PS-2, of Drosophila funebris. J. Insect Physiol. 20: 2181-2194.

- 1974b. Biological effects of paragonial substances PS-1 and PS-2 in females of Drosophila funebris. I. Insect Physiol. 20: $2347-2362$.

Baumann, H., K.J. Wilson, P.S. Chen, and R.E. Humbel. 1975. The amino-acid sequence of a peptide (PS-1) from Drosophila funebris: A paragonial peptide from males which reduces the receptivity of the females. Eur. I. Biochem. 52: $521-529$.

Belote, J.M. and B.S. Baker. 1982. Sex determination in Drosophila melanogaster: Analysis of transformer-2, a sex-transforming locus. Proc. Natl. Acad. Sci. 79: 1568-1572.

Birnstiel, M.L., M. Busslinger, and K. Strub. 1985. Transcription termination and $3^{\prime}$ end processing: The end is in site! Cell 41: 349-359.

Boswell, R.E. and A.P. Mahowald. 1985. tudor, a gene required for assembly of the germ plasm in Drosophila melanogaster. Cell 43: 97-104.

Bowtell, D.D.L., M.A. Simon, and G.M. Rubin. 1988. Nucleotide sequence and structure of the sevenless gene of Drosophila melanogaster. Genes Dev. 2: 620-634.

Breathnach, R. and P. Chambon. 1981. Organization and expression of eucaryotic split genes coding for proteins. Annu. Rev. Biochem. 50: 349-383.

Chapman, K.B. and M.F. Wolfner. 1988. Determination of male-specific gene expression in Drosophila accessory glands. Dev. Biol. 126: 195-202.

Chen, C.-N., S. Denome, and R.L. Davis. 1986. Molecular analysis of cDNA clones and the corresponding genomic coding sequences of the Drosophila dunce + gene, the structural gene for cAMP phosphodiesterase. Proc. Natl. Acad. Sci. 83: 9313-9317.

Chen, P.S. 1984. The functional morphology and biochemistry of insect male accessory glands and their secretions. Annu. Rev. Entomol. 29: 233-255.

Chen, P.S. and R. Bühler. 1970. Paragonial substance (sex peptide) and other free ninhydrin-positive components in male and female adults of Drosophila melanogaster. I. Insect Physiol. 16: 615-627.

Chen, P.S., E. Stumm-Zollinger, and M. Caldelari. 1985. Protein metabolism of Drosophila melanogaster male accessory glands II. Species-specificity of secretion proteins. Insect Biochem. 15: 385-390.

Chiu, A.Y., M.W. Hunkapiller, E. Heller, D.K. Stuart, L.E. Hood, and F. Strumwasser. 1979. Purification and primary structure of the neuropeptide egg-laying hormone of Aplysia californica. Proc. Natl. Acad. Sci. 76: 6656-6660.

DiBenedetto, A., D.M. Lakich, W.D. Kruger, J.M. Belote, B.S. Baker, and M.F. Wolfner. 1987. Sequences expressed sex-specifically in Drosophila melanogaster adults. Dev. Biol. 119: $242-251$.

Federer, H. and P.S. Chen. 1982. Ultrastructure and nature of secretory proteins in the male accessory gland of Drosophila funebris. I. Insect Physiol. 28: 743-751. 
Fitch, W.M. 1966. An improved method of testing for evolutionary homology. J. Mol. Biol. 16: 9-16.

Fowler, G.L. 1973. Some aspects of the reproductive biology of Drosophila: sperm transfer, sperm storage, and sperm utilization. Adv. Genet. 17: 293-360.

Garcia-Bellido, A. 1964. Das sekret der paragonien als stimulus der fekundität bei weibchen von Drosophila melanogaster. Z. Naturf. 19b: 491-495.

Giesow, M.J. and D.G. Smyth. 1980. Proteolysis of prohormones and proproteins. In The enzymology of post-translational modification of proteins, (ed. R.B. Freedman and H.C. Hawkins) vol. 1, pp. 259-287. Academic Press, London.

Gross, R.H. 1986. A DNA sequence analysis program for the Apple Macintosh. Nucleic Acids Res. 14: 591-596.

Guo, L.-H., P.P. Stepien, J.Y. Tso, R. Brousseau, S. Narang, D.Y. Thomas, and R. Wu. 1984. Synthesis of human insulin gene VIII. Construction of expression vectors for fused proinsulin production in Escherichia coli. Gene 29: 251-254.

Harwood, R. 1980. Protein transfer across membranes: the role of signal seqeunces and signal peptidase activity. In The enzymology of post-translational modification of proteins (ed. R.B. Freedman and H.C. Hawkins) vol. 1, pp. 3-52. Academic Press, London.

Henikoff, S. 1984. Unidirectional digestion with exonuclease III creates targeted breakpoints for DNA sequencing. Gene 28: $351-359$.

Hopp, T.P. and K.R. Woods. 1981. Prediction of protein antigenic determinants from amino acid sequence. Proc. Natl. Acad. Sci. 78: 3824-3828.

Ingman-Baker, J. and E.P.M. Candido. 1980. Proteins of the Drosophila melanogaster male reproductive system: Twodimensional gel patterns of proteins synthesized in the $\mathrm{XO}$, $X Y$, and XYY testis and paragonial gland and evidence that the $\mathrm{Y}$ chromosome does not code for structural sperm proteins. Biochem. Genet. 18: 809-828.

Kaldany, R.-R.J., J.R. Nambu, and R.H. Scheller. 1985. Neuropeptides in identified Aplysia neurons. Annu. Rev. Neurosci. 8: 431-455.

Komalski, D., W.D. Kroeker, M. Laskowski, Sr. 1976. Mung bean nuclease I. Terminally directed hydrolysis of native DNA. Biochem. 15: 4463-4467.

Kornfeld, R. and S. Kornfeld. 1985. Assembly of asparaginelinked oligosaccharides. Annu. Rev. Biochem 54: 631-664.

Kurjan, J. and I. Herskowitz. 1982. Structure of a yeast pheromone gene $(M F \alpha)$ : A putative $\alpha$-factor precursor contains four tandem repeats of mature $\alpha$-factor. Cell 30: 933-943.

Laemmli, U.K. 1970. Cleavage of structural proteins during the assembly of the head of bacteriophage T4. Nature 227: 680685.

Leopold, R.A. 1976. The role of male accessory glands in insect reproduction. Annu. Rev. Entomol. 21: 199-221.

MacIntyre, R.J. and M.B. Davis. 1987. A genetic and molecular analysis of the $\alpha$-glycerophosphate cycle in Drosophila me lanogaster. In Isozymes: Current topics in biological and medical research, 14: molecular and cellular biology, led. M.C. Rattazzi, J.G. Scandalios, and G.S. Whitel, pp. 195-227. Alan R. Liss, New York.

Maniatis, T., E.F. Fritsch, and J. Sambrook. 1982. Molecular cloning: A laboratory manual. Cold Spring Harbor Laboratory, Cold Spring Harbor, New York.

Manning, A. 1962. A sperm factor affecting the receptivity of Drosophila melanogaster females. Nature 194: 252-253.

Mizusawa, S., S. Nishimura, and F. Seela. 1986. Improvement of the dideoxy chain termination method of DNA sequencing by use of deoxy-7-deazaguanosine triphosphate in place of dGTP. Nucleic Acids Res. 14: 1319-1324.
Mlodzik, M. and W.J. Gehring. 1987. Expression of the caudal gene in the germ line of Drosophila: Formation of an RNA and protein gradient during early embryogenesis. Cell 48: 465-478.

Mount, S. 1982. A catalogue of splice junction sequences. $\mathrm{Nu}$ cleic Acids Res. 10: 459-472.

Nambu, J.R. and R.H. Scheller. 1986. Egg-laying hormone genes of Aplysia: Evolution of the ELH gene family. I. Neurosci. 6: 2026-2036.

Reinke, R., D.E. Krantz, D. Yen, and S.L. Zipursky. 1988. Chaoptin, a cell surface glycoprotein required for Drosophila photoreceptor cell morphogenesis, contains a repeat motif found in yeast and man. Cell 52: 291-301.

Sanger, G., S. Nicklen, A.R. Coulson. 1977. DNA sequencing with chain-terminating inhibitors. Proc. Natl. Acad. Sci. 74: $5463-5467$.

Schäfer, U. 1986. The regulation of male-specific transcripts by sex-determining genes in Drosophila melanogaster. EMBO J. 5: 3579-3582.

Scheller, R.H., J.F. Jackson, L.B. McAllister, B.S. Rothman, E. Mayeri, and R. Axel. 1983. A single gene encodes multiple neuropeptides mediating a stereotyped behavior. Cell 32: $7-22$.

Scholnick, S.B., S.J. Bray, B.A. Morgan, C.A. McCormick, and J. Hirsh. 1987. CNS and hypoderm regulatory elements of the Drosophila Dopa decarboxylase gene. Science 234: 9981002.

Stumm-Zollinger, E. and P.S. Chen. 1985. Protein metabolism of Drosophila melanogaster male accessory glands. I. Characterization of secretory proteins. Insect Biochem. 15: 375383.

Sun, X.-H., J.T. Lis, and R. Wu. 1988. The positive and negative transcriptional regulation of the Drosophila GAPDH-2 gene. Genes Dev. 2: 743-753.

Towbin, J., T. Staehelin, and J. Gordon. 1979. Electrophoretic transfer of proteins from polyacrylamide gels to nitrocellulose sheets: procedure and some applications. Proc. Natl. Acad. Sci. 76: 4350-4354.

von Heijne, G. 1983. Patterns of amino acids near signal-sequence cleavage sites. Eur. J. Biochem. 133: 17-21.

Whalen, M. and T.G. Wilson. 1986. Variation and genomic localization of genes encoding Drosophila melanogaster male accessory gland proteins separated by sodium dodecyl sulfate-polyacrylamide gel electrophoresis. Genetics 114: 7792. 


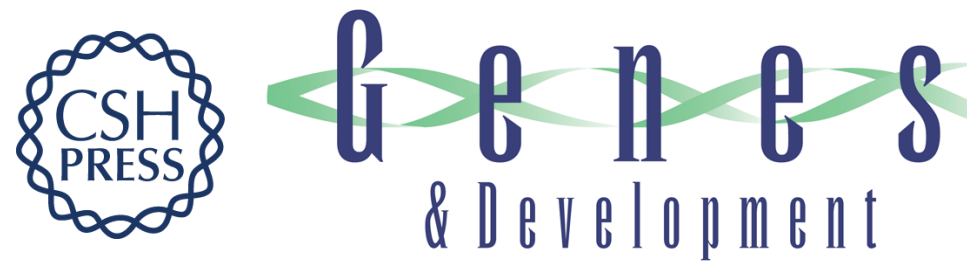

\section{Structure and expression of a Drosophila male accessory gland gene whose product resembles a peptide pheromone precursor.}

S A Monsma and M F Wolfner

Genes Dev. 1988, 2:

Access the most recent version at doi:10.1101/gad.2.9.1063

References This article cites 46 articles, 10 of which can be accessed free at: http://genesdev.cshlp.org/content/2/9/1063.full.html\#ref-list-1

License

Email Alerting

Service

Receive free email alerts when new articles cite this article - sign up in the box at the top right corner of the article or click here.

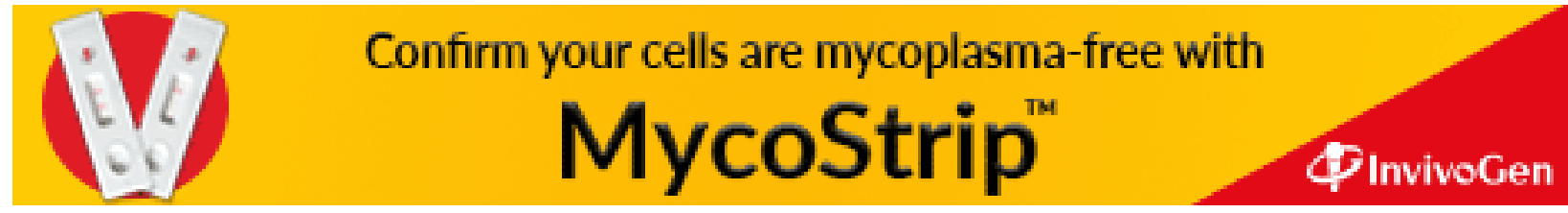

